\title{
Ratchet universality in the presence of thermal noise
}

\author{
Pedro J. Martínez ${ }^{1}$ and Ricardo Chacón ${ }^{2}$ \\ ${ }^{1}$ Departamento de Física Aplicada, E.I.N.A., Universidad de Zaragoza, E-50018 Zaragoza \\ and Instituto de Ciencia de Materiales de Aragón, CSIC-Universidad de Zaragoza, E-50009 Zaragoza, Spain \\ ${ }^{2}$ Departamento de Física Aplicada, Escuela de Ingenierías Industriales, Universidad de Extremadura, \\ Apartado Postal 382, E-06006 Badajoz, Spain, EU
}

(Received 16 November 2012; revised manuscript received 3 April 2013; published 10 June 2013)

\begin{abstract}
We show that directed ratchet transport of a driven overdamped Brownian particle subjected to a spatially periodic and symmetric potential can be reliably controlled by tailoring a biharmonic temporal force, in coherence with the degree-of-symmetry-breaking mechanism. We demonstrate that the effect of finite temperature on the purely deterministic ratchet scenario can be understood as an effective noise-induced change of the potential barrier which is in turn controlled by the degree-of-symmetry-breaking mechanism. Remarkably, we find that the same universal scenario holds for any symmetric periodic potential, while optimal directed ratchet transport occurs when the impulse transmitted (spatial integral over a half period) by the symmetric spatial force is maximum.
\end{abstract}

DOI: 10.1103/PhysRevE.87.062114

PACS number(s): 05.60.Cd, 05.40.-a, 05.70.Ln, 07.10.Cm

Directed transport without any net external force, the ratchet effect [1-3], has been an intensely studied interdisciplinary subject over the last few decades owing to its relevance in biology where ratchet mechanisms are found to underlie the working principles of molecular motors [4,5], and to its wide range of potential technological applications including microand nanotechnologies. Directed ratchet transport (DRT) is today understood to be a result of the interplay of symmetry breaking [6], nonlinearity, and nonequilibrium fluctuations in which these fluctuations include temporal noise [2], spatial disorder [7], and quenched temporal disorder [8]. Ratchets involving asymmetric substrates in the presence of noise are termed Brownian motors. The control of DRT in such systems is often limited by the impossibility of tuning the periodic substrate. However, when the periodic substrate is fixed, even symmetric under reflection, an effective way to yield and control DRT consists in driving it by means of an asymmetric time-periodic excitation, the simplest choice being the case of a biharmonic excitation (harmonic mixing). This technique has been applied in the context of nanoparticle transport as well as to design ring gyroscopes and annular Josephson junctions (see, e.g., Ref. [3] and references therein). In extremely small systems, including many of those occurring in biological and liquid environments as well as many nanoscale devices, DRT is often suitably described by overdamped ratchets, in which inertial effects are negligible in comparison with friction effects [2,9-11]. Here, we show how ratchet universality [12] works subtly in the context of noisy overdamped ratchets by studying the dynamics of a universal model-a Brownian particle moving on a periodic substrate subjected to a biharmonic excitation $[2,3]$,

$$
\begin{aligned}
\dot{x}+\sin x & =\sqrt{\sigma} \xi(t)+\gamma F_{\text {bihar }}(t), \\
F_{\text {bihar }}(t) & \equiv \eta \sin (\omega t)+2(1-\eta) \sin (2 \omega t+\varphi),
\end{aligned}
$$

where $\gamma$ is an amplitude factor, and the parameters $\eta \in[0,1]$ and $\varphi$ account for the relative amplitude and initial phase difference of the two harmonics, respectively, while $\xi(t)$ is a Gaussian white noise with zero mean and $\langle\xi(t) \xi(t+s)\rangle=$ $\delta(s)$, and $\sigma=2 k_{b} T$ with $k_{b}$ and $T$ being the Boltzmann constant and temperature, respectively. For deterministic ratchets, this has been shown to also be the case for topological solitons [8] and matter-wave solitons [13]. It is worth noting that, in spite of the abundance of numerical findings, the theoretical understanding of the directed transport phenomena represented by Eq. (1) remains far from being satisfactory [14] even about half a century after the earliest studies [15-17]. Indeed, all the earlier theoretical predictions (cf. Refs. [3,6,16-18]) indicate that the dependence of DRT velocity on the amplitudes of the two harmonics should scale as

$$
v \sim \gamma^{2} \eta^{2}(1-\eta) .
$$

Note that this expression presents, as a function of $\eta$, a single maximum at $\eta=2 / 3$, irrespective of the particular value of temperature, including the limiting value $T=0$ (cf. Refs. [3,18]). The occurrence of DRT in Eq. (1) implies the breakage of two temporal symmetries: the shift symmetry and the time-reversal symmetry of the biharmonic excitation [2]. For deterministic ratchets subjected to biharmonic forces, it has been shown [12] that there exists a universal force waveform which optimally enhances directed transport by symmetry breaking. Specifically, such a particular waveform has been shown to be unique for both temporal and spatial biharmonic forces. This universal waveform is a direct consequence of the degree of symmetry breaking (DSB) mechanism: It is possible to consider a quantitative measure of the DSB on which the strength of directed transport by symmetry breaking must depend. This mechanism has led to the unveiling of a criticality scenario for DRT. Indeed, it has been shown that optimal enhancement of DRT is achieved when maximal effective (i.e., critical) symmetry breaking occurs, which is in turn a consequence of two reshaping-induced competing effects: the increase of the DSB and the decrease of the (normalized) maximal transmitted impulse over a half-period, thus implying the existence of a particular force waveform which optimally enhances DRT (see [12] for additional details). Since thermal noise is significant in magnitude and unavoidable in diverse physical contexts, the following question naturally arises: How does the DSB mechanism work at finite temperatures? 
In the present work, we shall address this important question and provide analytical estimates for the dependence of the DRT on the system's parameters which are in excellent agreement with numerical results. To study numerically the effect of thermal noise $(\sigma>0)$ on the purely deterministic ratchet scenario, we calculated the mean velocity on averaging over different realizations of noise $\langle\langle\dot{x}\rangle\rangle \equiv v$ [cf. Eq. (1)]. Since Gaussian white noise does not break any relevant symmetry of Eq. (1), and the ratchet universality [12] predicts (for $\sigma=0$ ) that the optimal value of the relative amplitude $\eta$ comes from the condition that the amplitude of $\sin (\omega t)$ must be twice as large as that of $\sin (2 \omega t+\varphi)$ in Eq. (1), naïvely, one might expect that the average velocity should present, as a function of $\eta$, a single maximum at $\eta=\eta_{o p t} \equiv 4 / 5$ when $\varphi \neq 0, \pi$, as in the purely deterministic case. However, our numerical estimates of the $\eta$ value at which the average velocity is maximum, $\eta_{\text {opt }}^{\sigma>0}$, indicate a systematic deviation from $\eta_{o p t} \equiv$ 4/5: $\Delta \eta \equiv 4 / 5-\eta_{o p t}^{\sigma>0}>0$, which is independent of the noise intensity over a significant finite range, as is shown in Figs. 1(a) and 2 . Note that these results are at variance with the prediction from Eq. (2) (i.e., $\eta_{\text {opt }} \equiv 2 / 3$ ). To explain this paradox, the following remarks are in order. First, the effect of noise on the DRT strength depends on the amplitude of the biharmonic excitation while keeping the remaining parameters constant. Second, while changing $\eta$ and $\varphi$ allows one to control the breakage of the aforementioned relevant symmetries, it also changes the amplitude of the biharmonic excitation. Since the strength of any transport (whether or not induced by symmetry breaking) depends on the amplitude of the driving excitation, one concludes that these two effects of changing $\eta$ or $\varphi$ overlap, so that one will find it difficult to distinguish the contribution to transport that is purely due to symmetry breaking, and hence to clarify the interplay between noise and symmetry breaking. We shall therefore consider an affine transformation of the biharmonic excitation $F_{\text {bihar }}(t)$, for the optimal value $\varphi=\varphi_{o p t} \equiv \pi / 2$, for example [19], to change its image to $[-1 / 2,1 / 2], \forall \eta$, thus making it possible to characterize the genuine effect of noise on the purely deterministic ratchet scenario:

$$
f_{\varphi=\pi / 2}^{*}(t) \equiv \frac{F_{\text {bihar }}(t)-m}{M-m}-\frac{1}{2},
$$

where $m=m(\eta) \equiv \eta-2, \forall \eta$, while $M=M(\eta) \equiv \frac{\eta^{2}+32(1-\eta)^{2}}{16(1-\eta)}$ for $0 \leqslant \eta \leqslant 8 / 9$ and $M(\eta) \equiv 3 \eta-2$ for $8 / 9 \leqslant \eta \leqslant 1$ [see Fig. 1(b)]. After substituting Eq. (3) into Eq. (1), one straightforwardly obtains

$$
\frac{d x}{d t^{\prime}}+\frac{1}{\Lambda} \sin x=\gamma W+\gamma f_{\varphi=\pi / 2}^{*}\left(t^{\prime}\right)+\sqrt{\sigma^{\prime}} \xi\left(t^{\prime}\right),
$$

where $\Lambda=\Lambda(\eta) \equiv M-m, t^{\prime}=t^{\prime}(t, \eta) \equiv \Lambda t, W=W(\eta) \equiv$ $(M+m) /(2 \Lambda), \omega^{\prime}=\omega^{\prime}(\omega, \eta) \equiv \omega / \Lambda$, and $\sigma^{\prime}=\sigma^{\prime}(\sigma, \eta) \equiv$ $\sigma / \Lambda$. It is worth noting that the function $\Lambda(\eta)$ is merely the width of the image of $F_{\text {bihar }}(t)$, i.e., the difference between its maxima and minima as a function of $\eta$, and that it presents a single minimum at $\eta=\eta^{*} \equiv 6 / 7$. Also, the function $W(\eta)$ represents an $\eta$-dependent "load" force having a single maximum (in absolute value) at $\eta=\eta_{\text {opt }} \equiv 4 / 5$, while $W(\eta=0,1)=0$, as expected. These particular values of $\eta^{*}$ and $\eta_{\text {opt }}$ are a direct consequence of the application of ratchet universality to the specific form of the present biharmonic
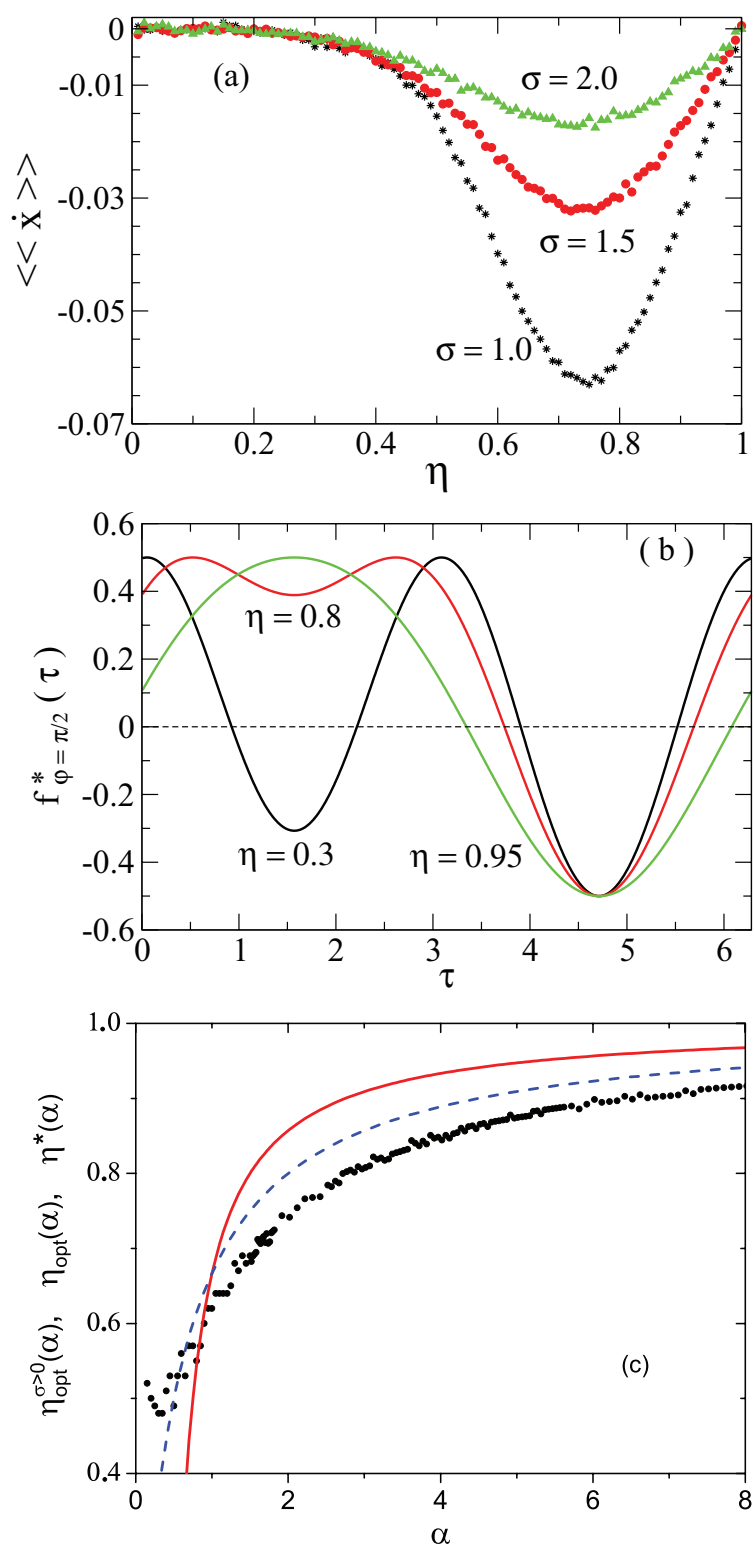

FIG. 1. (Color online) (a) Average velocity $\langle\langle\dot{x}\rangle\rangle \equiv\left\langle\left\langle\Lambda d x / d t^{\prime}\right\rangle\right\rangle$ [cf. Eqs. (1) and (4)] versus relative amplitude $\eta$ for $\varphi=\varphi_{\text {opt }} \equiv$ $\pi / 2, \omega=0.08 \pi, \gamma=2$, and three values of the noise intensity. (b) Normalized biharmonic function [Eq. (3)] versus time for $\omega=1$ and three values of $\eta$. (c) Value of $\eta$ where the average velocity is maximum, $\eta_{o p t}^{\sigma>0}$, versus $\alpha$ [cf. Eq. (1) with $F_{b i h a r}^{\prime}(t)$ instead of $F_{\text {bihar }}(t)$; see the text] for $\varphi=\varphi_{\text {opt }} \equiv \pi / 2, \omega=0.08 \pi, \gamma=2$, and $\sigma=1$. Also plotted is the theoretical prediction for the purely deterministic case $\eta_{\text {opt }}(\alpha) \equiv 2 \alpha /(1+2 \alpha)$ (dashed line) and the function $\eta^{*}(\alpha) \equiv(4 \alpha-2) /(4 \alpha-1)$ (see the text; solid line).

excitation $F_{\text {bihar }}(t)$. However, to better understand the roots of the present problem, it is convenient to consider the more general form $F_{b i h a r}^{\prime}(t) \equiv \eta \sin (\omega t)+\alpha(1-\eta) \sin (2 \omega t+\varphi)$, with $\alpha>0$ being a parameter. For this case, one has $\eta^{*}=\eta^{*}(\alpha) \equiv(4 \alpha-2) /(4 \alpha-1), \eta_{o p t}=\eta_{o p t}(\alpha) \equiv 2 \alpha /(1+$ $2 \alpha)$, while the deviation $\Delta \eta(\alpha) \equiv \eta_{\text {opt }}(\alpha)-\eta_{\text {opt }}^{\sigma>0}(\alpha)$ suggests a certain correlation between $\eta_{\text {opt }}(\alpha)$ and $\eta_{\text {opt }}^{\sigma>0}(\alpha)$ over a wide range of $\alpha$ values from $\alpha \simeq 2$ [see Fig. 1(c)]. Once again, all the earlier theoretical analysis (cf. Refs. [3,6,16-18]) predict 

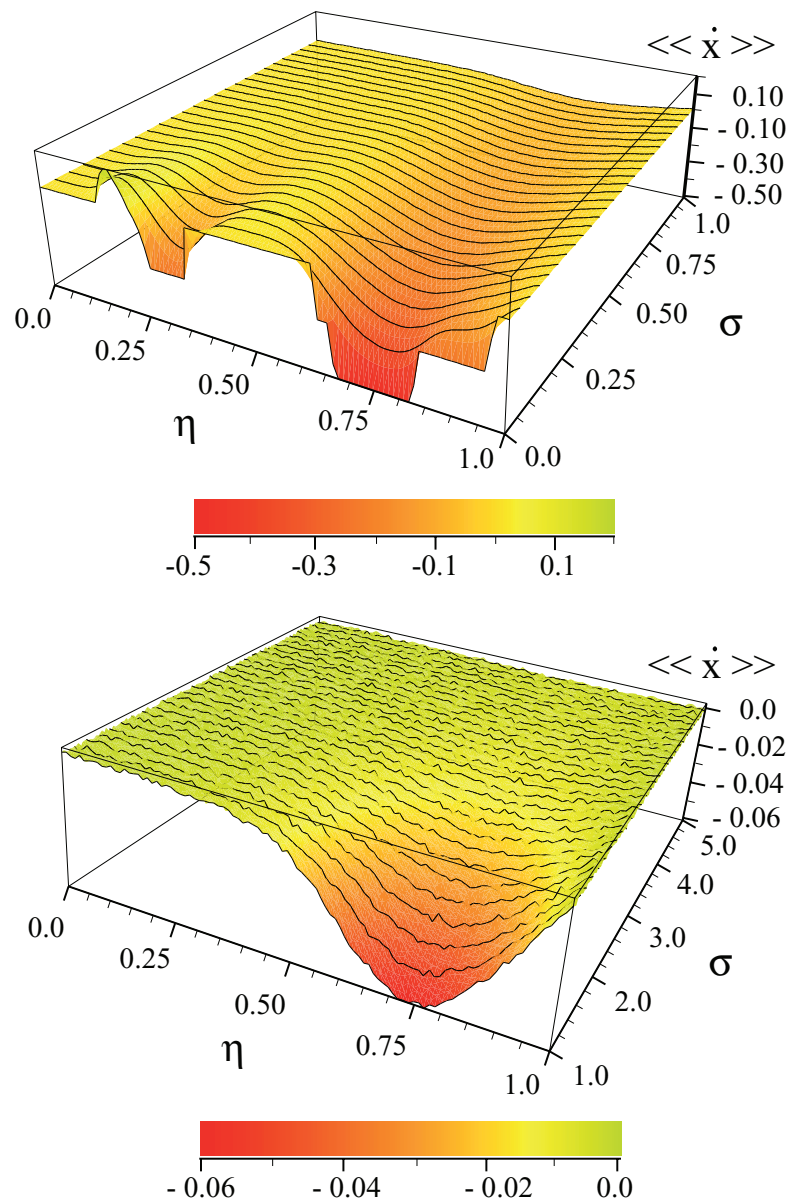

FIG. 2. (Color online) Average velocity $\langle\langle\dot{x}\rangle\rangle$ [cf. Eq. (1)] versus relative amplitude $\eta$ and noise intensity $\sigma$ for $\varphi=\varphi_{\text {opt }} \equiv \pi / 2, \omega=$ $0.08 \pi$, and $\gamma=2$. The ranges of small and large noise intensities are shown in the top and bottom panels, respectively.

[for $F_{b i h a r}^{\prime}(t)$ ] that the average velocity should present, as a function of $\eta$, a single maximum at $\eta=2 / 3$, irrespective of the particular values of temperature (including the limiting value $T=0$ ) and parameter $\alpha$. Together, these results therefore allow one to draw the following conclusions from Eq. (4).

First, the aforementioned twofold transport effect of a biharmonic excitation $F_{\text {bihar }}(t)$, as $\eta$ varies from 0 to 1 , may be decoupled into two terms: a constant excitation, $W$, and a biharmonic excitation, $f_{\varphi=\pi / 2}^{*}\left(t^{\prime}\right)$, having an amplitude which is independent of $\eta$. The relevant observation is that both excitations yield a maximum strength of transport at $\eta=\eta_{o p t} \equiv 4 / 5$. Therefore, replacing $F_{\text {bihar }}(t)$ with $f_{\varphi=\pi / 2}^{*}(t)$ in Eq. (1),

$$
\dot{x}+\sin x=\sqrt{\sigma} \xi(t)+\gamma f_{\varphi=\pi / 2}^{*}(t)
$$

should yield a maximum average velocity at $\eta=\eta_{\text {opt }} \equiv 4 / 5$, as is indeed confirmed by numerical experiments (see Fig. 3). Thus, we propose for the system (5) the following scaling for the average velocity:

$$
\langle\langle\dot{x}\rangle\rangle \sim C W(\eta)
$$

where $C$ is a fitting constant that depends on the remaining system parameters. Furthermore, for sufficiently high temperature (i.e., sufficiently far from the "steps" regime occurring at

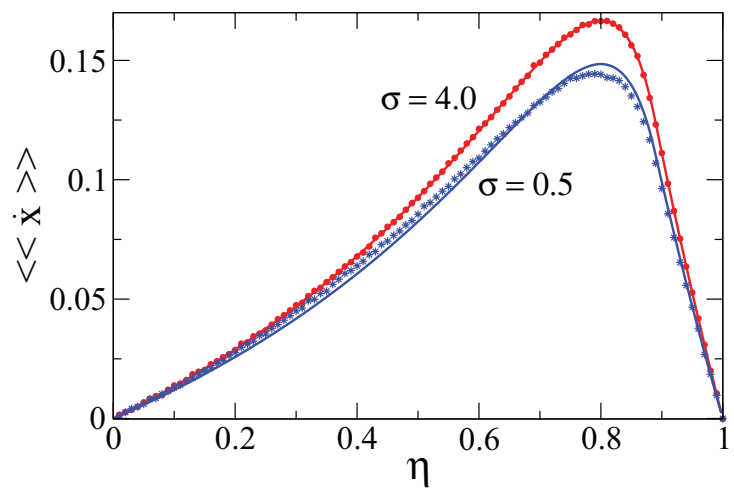

FIG. 3. (Color online) Average velocity $\langle\langle\dot{x}\rangle\rangle$ [cf. Eq. (5)] versus relative amplitude $\eta$ for $\omega=0.08 \pi, \gamma=2$, and two values of the noise intensity. Also plotted is the scaling law (6) for $C=1.00017$ ( $\sigma=4)$ and $C=0.89072(\sigma=0.5)$ [cf. Eq. (6); solid lines].

$\sigma=0$; see Fig. 2) and driving amplitude (i.e., in the absence of stochastic resonance effects), exact agreement between numerical results and scaling (6) is expected over the complete range of $\eta$ values (see Fig. 3), while the scaling (6) remains valid over a wide range of frequencies (data not shown).

And second, alternatively to the case discussed in the first conclusion, only rescaling the temperature with the width $\Lambda$ in Eq. (1) in accordance with Eq. (4),

$$
\dot{x}+\sin x=\sqrt{\Lambda \sigma} \xi(t)+\gamma F_{b i h a r}(t)
$$

should also yield a maximum average velocity at $\eta=\eta_{\text {opt }} \equiv$ 4/5. Numerical experiments also confirmed this prediction, as in the illustrative examples shown in Fig. 4(a). Thus, it is only after rescaling $\sigma \rightarrow \Lambda \sigma$ in Eq. (1) that one recovers the purely deterministic ratchet scenario, which was an unanticipated result. To make the comparison between Eqs. (1) and (7) clearer, let us first transform Eq. (7) into the equation

$$
\begin{aligned}
\dot{x}+\frac{1}{\Lambda} \sin x= & \sqrt{\sigma} \xi(t)+\gamma^{\prime}\left[\eta \sin \left(\omega^{\prime} t\right)\right. \\
& \left.+2(1-\eta) \sin \left(2 \omega^{\prime} t+\varphi\right)\right]
\end{aligned}
$$

by rescaling the time, $t \rightarrow \Lambda t$, and where $\gamma^{\prime} \equiv \gamma / \Lambda, \omega^{\prime} \equiv$ $\omega / \Lambda$. Since the transport properties of Eq. (8) are similar to those of Eq. (7) in the sense that their respective average velocities present a single extremum at the same value of $\eta$ for a fixed set of the remaining parameters, and that such an optimal value, $\eta_{o p t} \equiv 4 / 5$, is independent of the particular values of the amplitude and the frequency of the biharmonic excitation [12], one concludes from the comparison of Eqs. (1) and (8) that the effect of thermal noise on the purely deterministic ratchet scenario can be understood as an effective noise-induced change of the potential barrier $[d=d(\eta) \equiv \Lambda(\eta)]$ which is in turn controlled by the degree-of-symmetry-breaking mechanism through the function $\Lambda(\eta)$. Recall that the average velocity $\langle\langle\dot{x}\rangle\rangle$ exhibits, as a function of the potential barrier $d$, a single maximum due to the thermal interwell activation (TIA) mechanism and the limiting behaviors $\lim _{d \rightarrow 0, \infty}\langle\langle\dot{x}\rangle\rangle=0$ [14]. Obviously, the same scenario also holds when $F_{b i h a r}(t)$ is replaced with $F_{b i h a r}^{\prime}(t)$, which allows one to understand the behavior of the deviation $\Delta \eta(\alpha) \equiv \eta_{\text {opt }}(\alpha)-\eta_{o p t}^{\sigma>0}(\alpha)$ as $\alpha$ is changed [cf. Fig. 1(c)]. Clearly, one can distinguish three regimes. Over the range $0<\alpha \lesssim 1 / 2$, the TIA mechanism 

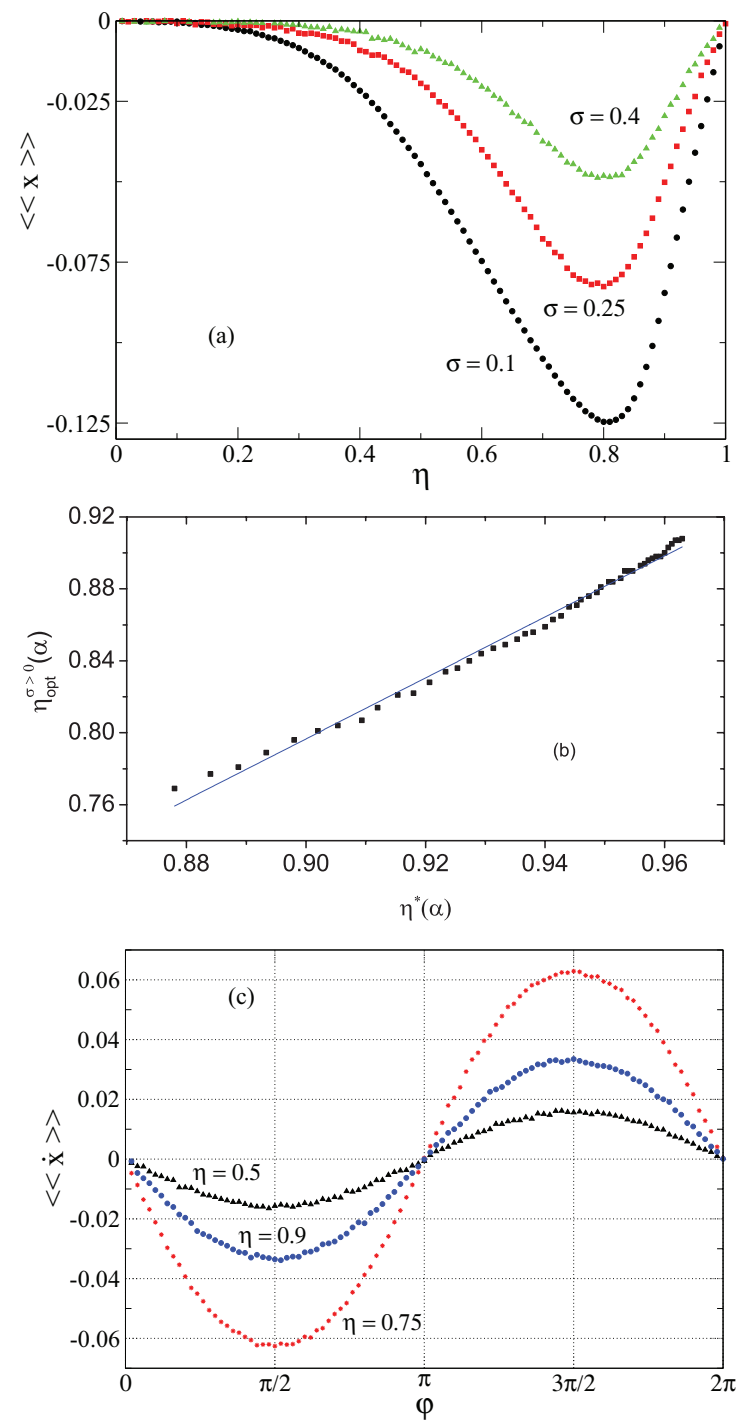

FIG. 4. (Color online) (a) Average velocity $\langle\langle\dot{x}\rangle\rangle$ [cf. Eq. (7)] versus relative amplitude $\eta$ for $\omega=0.08 \pi, \gamma=2$, and three values of the noise intensity. (b) Value of $\eta$ where the average velocity is maximum, $\eta_{o p t}^{\sigma>0}(\alpha)$, versus function $\eta^{*}(\alpha)$ [cf. Eq. (1) with $F_{b i h a r}^{\prime}(t)$ instead of $F_{\text {bihar }}(t)$; see the text] and linear fit (9) (solid line) over the range $2.3 \leqslant \alpha \leqslant 7$ for $\varphi=\varphi_{o p t} \equiv \pi / 2, \omega=0.08 \pi, \gamma=2$, and $\sigma=1$. (c) Average velocity $\langle\langle\dot{x}\rangle\rangle$ [cf. Eq. (1)] versus initial phase difference $\varphi$ for $\omega=0.08 \pi, \gamma=2, \sigma=1$, and three values of $\eta$.

dominates over the DSB mechanism. Indeed, the contribution of the DSB mechanism to directed transport becomes ever smaller as $\alpha \rightarrow 0$ because $\eta_{\text {opt }}(\alpha \rightarrow 0) \rightarrow 0$, and hence the (whole) amplitude of the biharmonic excitation for which the DRT strength is maximum also becomes ever smaller as $\alpha \rightarrow 0$, while the contribution of the TIA mechanism remains significant over the entire range $0<\alpha \lesssim 1 / 2$. Then one observes a transition regime over the range $1 / 2 \lesssim \alpha \lesssim 2$ as the effect of the DSB mechanism strengthens, which is manifest in the existence of a narrow range of $\alpha$ values in which $\Delta \eta(\alpha) \approx 0$ [see Fig. 1(c)]. Finally, for the range $\alpha \gtrsim 2$, the contributions of the DSB and TIA mechanisms are correlated in the sense of the aforementioned effective noise-induced change of the potential barrier. This means that $\eta_{o p t}^{\sigma>0}(\alpha)$ is expected to be proportional to the value of the relative amplitude where the effective noise-induced potential barrier presents a minimum:

$$
\eta_{\text {opt }}^{\sigma>0}(\alpha) \sim A \eta^{*}(\alpha)+B
$$

with $A \simeq 1.71, B \simeq-0.73$ being constants that are independent of the remaining system parameters. The general scaling (9) is confirmed by numerical experiments [see Fig. 4(b)]. Also, with regard to the dependence of the DRT strength on the initial phase difference, numerical results confirmed the scaling $\langle\langle\dot{x}\rangle\rangle \sim C^{\prime} \sin \varphi$, where $C^{\prime}$ is a fitting constant that depends on the remaining system parameters, in accordance with ratchet universality [12] [see Fig. 4(c)]. Finally, it is worth noting that the same scenario holds when the present sinusoidal potential $V(x) \equiv-\cos x$ [cf. Eq. (1)] is replaced with any symmetric (under reflection) periodic potential. For the sake of clarity, we show this property by considering the generalized model

$$
\dot{x}+\frac{\partial U(x ; a)}{\partial x}=\sqrt{\sigma} \xi(t)+\gamma F_{b i h a r}(t),
$$

where the spatial force $-\partial U(x ; a) / \partial x \equiv$ $-\tanh (a \sin x) / \tanh (a)$ has the same amplitude for any wave form (i.e., $\forall a \in[0, \infty])$. One has $[\partial U(x ; a) / \partial x]_{a=0}=\sin x$ while, in the other limit, $[\partial U(x ; a) / \partial x]_{a=\infty}$ is the square-wave function (see Fig. 5, top panel). We find that optimum DRT occurs when the impulse transmitted (spatial integral over a half period) by the spatial force is maximum while keeping the remaining parameters fixed, as is shown in Fig. 5 (bottom
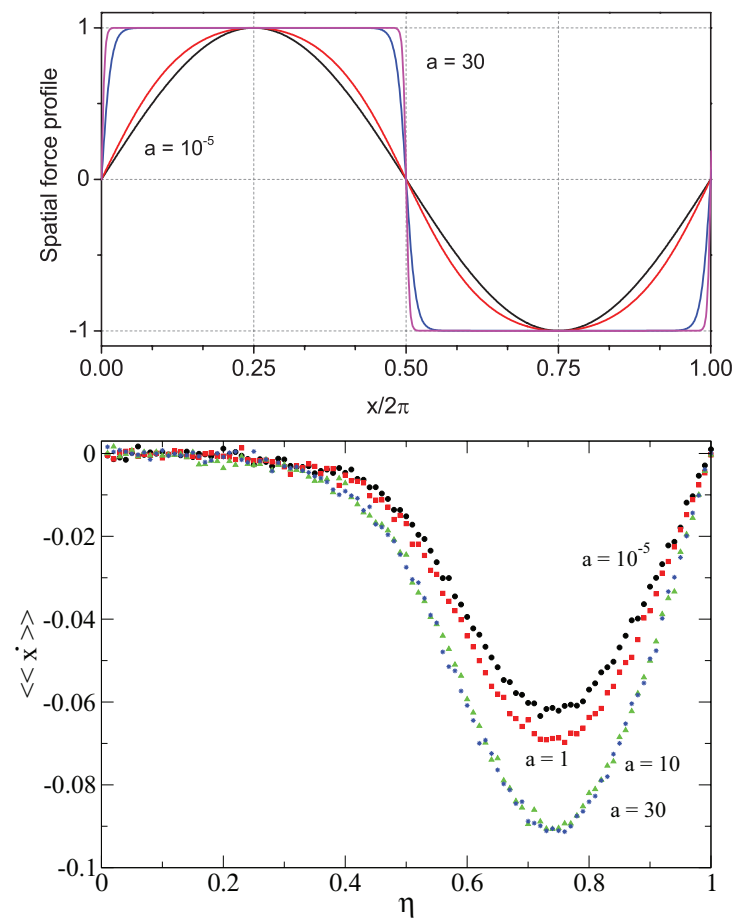

FIG. 5. (Color online) Top: Wave forms of the spatial force $-\tanh (a \sin x) / \tanh (a)$ for four values of the shape parameter: $a=$ $10^{-5}$ (nearly sinusoidal pulse), $a=1, a=10$, and $a=30$ (nearly square-wave pulse). Bottom: Average velocity $\langle\langle\dot{x}\rangle\rangle$ [cf. Eq. (10)] versus relative amplitude $\eta$ for $\varphi=\varphi_{\text {opt }} \equiv \pi / 2, \omega=0.08 \pi, \gamma=$ $2, \sigma=1$, and the same four values of the shape parameter $a$. 


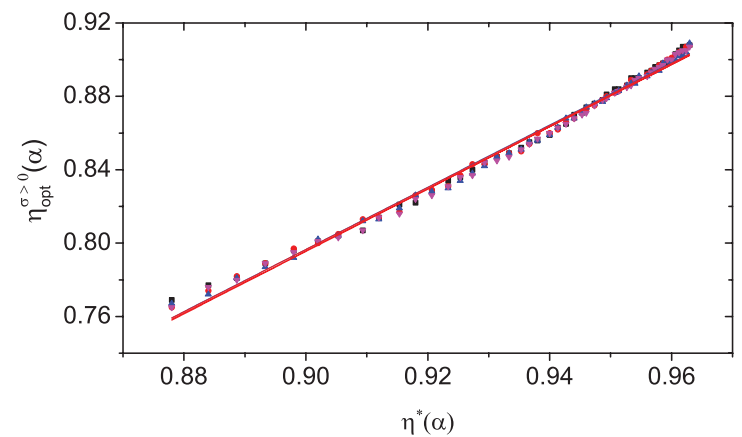

FIG. 6. (Color online) Value of $\eta$ where the average velocity is maximum, $\eta_{o p t}^{\sigma>0}(\alpha)$, versus function $\eta^{*}(\alpha)$ [cf. Eq. (10) with $F_{b i h a r}^{\prime}(t)$ instead of $F_{\text {bihar }}(t)$; see the text] and linear fit (9) (solid line) over the range $2.3 \leqslant \alpha \leqslant 7$ for $\varphi=\varphi_{o p t} \equiv \pi / 2, \omega=0.08 \pi, \gamma=2, \sigma=$ 1 , and four values of the shape parameter: $a=10^{-5}$ (circles), $a=1$ (squares), $a=10$ (triangles), and $a=30$ (stars).

panel). Remarkably, the general scaling (9) holds for any value of the shape parameter $a$ (see Fig. 6), thus confirming its universality [20].

In summary, we have explained the interplay between thermal noise and symmetry breaking in the ratchet transport of a Brownian particle moving on a periodic substrate subjected to a temporal biharmonic excitation, in coherence with the degree-of-symmetry-breaking mechanism. For any finite value of temperature, including the deterministic limit $(T=0)$, the reason of the failure of all the earlier theoretical predictions [cf. Refs. [3,6,16-18], Eq. (2)] is now clear: Both the moment expansion method $[3,6,15]$ and the functional Taylor expansion formalism [18] assume that the contributions of the amplitudes of the two harmonics to the average velocity are independent. However, the existence of a universal waveform which optimally enhances directed ratchet transport [12] implies that the two amplitudes are correlated in the sense mentioned above. Thus, the general ratchet scenario presented in this work provides a reliable theoretical framework for the optimal control of the dynamics of Brownian ratchets in future applications, including dimers and more complex systems.

P.J.M. acknowledges financial support from the Ministerio de Ciencia e Innovación (MICINN, Spain) through Project No. FIS2011-25167 cofinanced by FEDER funds. R.C. acknowledges financial support from the MICINN, Spain, through Project No. FIS2012-34902 cofinanced by FEDER funds, and from the Junta de Extremadura (JEx, Spain) through Project No. GR10045.
[1] P. Hänggi and R. Bartussek, Lect. Notes Phys. 476, 294 (1996); special issue on Ratchets and Brownian Motors edited by H. Linke [Appl. Phys. A 75 (2002)].

[2] P. Reimann, Phys. Rep. 361, 57 (2002).

[3] P. Hänggi and F. Marchesoni, Rev. Mod. Phys. 81, 387 (2009).

[4] K. Sbovoda et al., Nature (London) 365, 721 (1993); D. Astumian, Science 276, 917 (1997).

[5] F. Jülicher, A. Ajdari, and J. Prost, Rev. Mod. Phys. 69, 1269 (1997).

[6] S. Flach, O. Yevtushenko, and Y. Zolotaryuk, Phys. Rev. Lett. 84, 2358 (2000); S. Denisov, S. Flach, A. A. Ovchinnikov, O. Yevtushenko, and Y. Zolotaryuk, Phys. Rev. E 66, 041104 (2002).

[7] A. B. Kolton, Phys. Rev. B 75, 020201(R) (2007).

[8] P. J. Martínez and R. Chacón, Phys. Rev. Lett. 100, 144101 (2008).

[9] G. Carapella and G. Costabile, Phys. Rev. Lett. 87, 077002 (2001).

[10] F. R. Alatriste and J. L. Mateos, Physica A 372, 263 (2006).

[11] R. Gommers, S. Denisov, and F. Renzoni, Phys. Rev. Lett. 96, 240604 (2006).
[12] R. Chacón, J. Phys. A: Math. Theor. 40, F413 (2007); 43, 322001 (2010).

[13] M. Rietmann, R. Carretero-González, and R. Chacón, Phys. Rev. A 83, 053617 (2011).

[14] M. Borromeo, P. Hänggi, and F. Marchesoni, J. Phys.: Condens. Matter 17, S3709 (2005); M. Borromeo and F. Marchesoni, Phys. Rev. E 73, 016142 (2006).

[15] W. Schneider and K. Seeger, Appl. Phys. Lett. 8, 133 (1966).

[16] W. Wonneberger and H. J. Breymayer, Z. Phys. B: Condens. Matter 43, 329 (1981).

[17] F. Marchesoni, Phys. Lett. A 119, 221 (1986).

[18] N. R. Quintero, J. A. Cuesta, and R. Alvarez-Nodarse, Phys. Rev. E 81, 030102(R) (2010).

[19] The analysis is quite similar for any other value of the initial phase difference $\varphi$. In particular, for the other optimal value, $\varphi_{\text {opt }} \equiv 3 \pi / 2$, one has DRT with the same strength but opposite direction to that corresponding to $\varphi_{\text {opt }} \equiv \pi / 2$ for each value of $\eta \in$ ]0,1[ (cf. Ref. [12]).

[20] The same excellent agreement with scaling (9) was found for other alternative symmetric spatial potentials (data not shown). 